\title{
Novel Network Topology and Transporting Protocol for Data Collection from Large Scale Smart Electricity Meters
}

\author{
Zhou Feng ${ }^{1,2}$, Cheng Yingying ${ }^{1,2}, \mathrm{Xiao}_{\mathrm{Ji}}{ }^{1,2}$, Du Jie $\mathrm{Ji}^{1,2}$ \\ ${ }^{1}$ State Grid Chongqing Electric Power CO. Electric Power Research Institute Chongqing, China \\ ${ }^{2}$ Energy Measuring Instruments Performance Evaluation Laboratory Chongqing, China \\ email: zfocus@foxmail.com
}

Keywords: smart electricity meter; data collection; large scale; SNMP

Abstract. With the smart electricity meters heavy using, efficient and reliable network and transportation protocol are becoming more and more important to collect energy data from the meters. This paper proposed a new network topology for data collection from large amount resident energy meters. This topology could provide flexible access service to user and improve network scalability. This paper also proposed a new data transferring protocol based on SNMP. The messages are classified into three types and encapsulated into trap packets. With an add-on acknowledgement mechanism, the new protocol could guarantee the delivery of important messages and merge other messages. It improved the utilization of the data collection network.

\section{Preface}

In recent years, increasingly digitized industrial production and social life made high demands for the power grid reliability [1]. However, the traditional power grid cannot meet the actual needs of supply reliability in the digital society. For the distribution and residential electricity network, the level of information management is low. It is difficult to get efficient and real-time access to accurate information on residential electricity[2].It is capable of monitoring, protection and automatic optimization run, including smart meters, including its internal interconnect components [3].

In order to achieve timely and effective information acquisition and processing of residential electricity, intelligent use of electricity, and provide data for a variety of applications to support smart grid in order to achieve energy saving, it is needed to design and implement topologies for efficient and reliable smart meter data collection network, and for large-scale information gathering residents meter network transmission protocol.

\section{Meter data collection technology analysis}

Now part of the power company uses a remote meter reading system based on wireless network through power communication network, mobile communications network in real-time energy data and other information gathering reliable back, through the application of a system with intelligent analysis software, to achieve user of electricity amount of statistics, analysis and use of the user's state electricity consumption [4]. Optical fiber communication network in Japan, distribution automation system has been widely used to provide good conditions for the implementation of advanced metering system [5].Smart meters can store a variety of measurement values such as active power, reactive power, voltage, current, and energy such as electricity. You can also configure the communication module on the smart meter, two-way communication system access and data center for information exchange [6]. Data concentrators typically arranged on the power tower, substation or other electrical infrastructure, focus is the intersection of LAN and WAN backbone to connect the user equipment and data communication networks. Data concentrator data center can also be sent to the downstream meter relay and the user's command and information [7].

In order to obtain real-time load management and more accurate monitoring results and the status of non-intrusive load monitoring and analysis of users, the number you need to need to improve the collection of 2-5 times the number of weekly data collection will be up to 500 million times. Obviously 
this amount of data for use only power line carrier communication is too big, you need to transfer data in different ways to focus, and then using fiber backbone telecommunications operators or communications network to the data collection center / control center.

\section{Support for IPv6 and flexible access to the new network architecture}

There are two main technologies: power line carrier (PLC) and wireless transmission network [8]. Transmission power line carrier communication distance farther, the investment is relatively small, can be synchronized with the grid construction. However, the low transmission rate, small data transmission capacity, application range is more limited [8]. For residential customers' smart meter data acquisition and condition monitoring, the adoption of PLC technology, communication capacity is obviously insufficient, and mainly PLC is a multi-user shared network upstream bandwidth. On the other hand power line carrier technology insurmountable transformer, so still need to be addressed across the district substations and other issues before they can access the high-pressure side of the grid connection of the company's backbone data communications network, essentially no fundamental solution to a wide range of remote network access problem.

Therefore, we have designed a new access network architecture major consideration technical approach based on wireless network transmission. Transmission of wireless network is further divided into: Based on Zigbee technology, based on the 802.11 wireless RF technology and data transmission technology [8].

The new network access structure will generally cost ZigBee communication module installed in the resident meter, with its short-distance data collection, remote control and management. Considering the ZigBee signal transmission within the building block and other issues, but also the need for additional network coordinator ZigBee protocol in section 802.11 pairs smart meter smart meter box at residents or outdoors (as a primary data concentrator). Floor building of shielding radio signal more serious, and the arrangement of the actual test and experience also shows that it is difficult to transmit directly to the interval of 5 floors dataset ZigBee device via the communication module, thus setting a planned every five layer 802.11 Primary data concentrator. Considering the focus has Type 2 way access to the backbone network, and therefore need to seriously consider its placement position. For direct access to the wired backbone 802.11 concentrator, the location should be convenient for the cable construction. For other concentrator through 802.11 access or trunk, you need to select the location of the wireless signal interference less, according to the channel conditions of nearby residents and users of WLAN equipment for home use, as far as possible to select its different non-overlapping channels. This will ensure the plurality of primary data concentrator 802.11 interconnected, but also to avoid the interference of the user's home wireless Internet services.

Such a multi-level access methods have the following advantages: 1. primary focus is to become the backbone of the communication mode with the gateway concentrator communication mode metering device. This makes the backbone network changes, affecting the communication interface module and the metering device is almost negligible. For example, the initial backbone network is leased telecommunications carrier's wireless WAN connection for each concentrator, post their own power companies to build a fiber-optic backbone network, you can only modify the network configuration and replace part of the backbone network interface concentrator.

On the other hand, the network communication mode metering device to modify or replace the device has no effect on the backbone. For example, some users upgrading to a table cell, the primary mode of communication by ZigBee centralized area to power line carrier communication. Simply replace the primary concentrator or upgraded to support power line carrier, do not need to change the primary focus and backbone networks. You can also use different devices on different network technologies, local conditions, and lower overall costs.

The price for one Zigbee module is only tens of dollars, the primary focus is the thousand-level price, the price is about the backbone concentrator million. Therefore, the communication equipment costs above cell collection network of about 20-30 million, taking into account the design life of the 
equipment for 8-10 years, only 2-3 million annual cost of equipment, household equipment costs as low as a few dozen yuan, much lower than the cost of manual meter reading, you can also get the load data and grid reliability data, and further remote load management.

\section{SNMP-based metering information collection protocol}

After the above flexible and efficient access to smart meter network construction, the need to design a simple and efficient, reliable operation of the smart meter to meter data transmission protocol state electricity transmission network information. This requires having a reliable transport protocol on the one hand, on the part of key information collection was confirmed; on the other hand to get general information gathering minimize network bandwidth and other resources, in order to avoid data overload significantly affect network performance or increase network the cost of construction.

Specific mechanisms are: measurement information will be in accordance with a fixed time interval to send by polling; and will change the status of the possible faults and other anomalies as Trap to send. But just such distinctions still have some problems. The key is the amount of meter is too great, and the short collection cycle, the amount of data. On the other hand, due to the change of state and the state of large-scale data analysis needs change frequently, bring a large amount of data, so that the primary focus is the transmission network is busy.

Through the nature and role of electricity metering and other data analysis can know, different data reliability requirements are different, and priorities are different. This brings us may utilize network transmission capacity, reduce the number Trap, the number of compressed packets transmitted poll. The key is to classify the merger mechanism Trap packets. First Trap packets as needed for data acquisition and device states the importance of classification. Then the importance of not high Trap messages are not sent and processed immediately when the message is generated. And a delay preset time interval. The importance of collecting data for the non-Trap messages within this interval were merged. Each PDU includes only a Trap message in the following format: time, and the trap again, this time within the segment generated combined sent together. With this classification and to delay non-critical messages sent and merge, greatly reduces the number of packets sent, reducing the impact of a large number of non-time-sensitive data transmission network resources.

Which PDU Type, Agent Addr other fields, from the same message a device is the same. Therefore, when the merger is sent, it only needs to be sent once, no need to repeat the information many times contain these fields, reducing unnecessary data network bandwidth and other resources. On the other hand, in order to make the receiver of the message data can be correctly resolved after the merger, the combined length of the Trap package also requires a small number of changes to the original message identifies the number of different and each message. Enables managers to split open a different trap original message is processed and stored. The specific method used is the use of the field in the original unused trap placement information, and modify variable bindings to indicate the location information of a different trap. The main processing flow modified as follows:

Step (1) in accordance with the measurement requirements, according to the remote control center energy measurement information and rules to determine the priority of the data and the data is classified according to priority. The charge fixed interval metering data classified as normal priority; the terminal alarm information classified as important information; the terminal failures and power-down state information classified as emergency information.

Step (2) the metering device has been classified report data into SNMP packets. First use LZW compression algorithm packet data is then packaged together with the priority information for SNMP packets compressed.

Step (3) adaptive information packets merged with transmission.

Step 3.1 metering device information collected through the network, to judge. If a general priority information, start the timer and go to step (3.4); otherwise go to step (3.2)

3.2 determine whether important information. If so, it sends a message, and requests the receiver transmit and receive acknowledgment, and then returns to the step (3A). Otherwise, go to step (3.3) 
3.3 send an emergency message.

3.4 determine whether the timer expires, if there is no overtime, turn back to the step (3.1). If the timeout before all general information into one overall report SNMP packets for transmission.

In the traditional trap in each message needs to be sent to the manager, the data part of the length of 86 bytes, the need to have an increase of 50-byte packet header information. For residential discussed above, if $5 \%$ of the information which events occur need to send trap, you need to generate about 500,000 trap messages per day. If direct transmission is $500000 * 136 \mathrm{~B}=68000000 \mathrm{~B}$, about680GB.

According to the actual needs of our experience and the information collected by the meter, it can be assumed with a message of the importance and timeliness of the request into three, and the proportion of emergency: General: No Emergency $=1: 2: 7$

You can increase recognition and urgent priority flag, generally do not change, not an emergency every 10 merge. This is not an emergency merger of 960-byte packets, the average available through the LZW algorithm compression $2 / 3$ packet, the packet is 320 bytes actually sent.

New data can be calculated by the number of transmitted data acquisition protocols:

500,000 divided into 50,000 packets of emergency, 100,000 general, 700,000 less urgent were merged into 70000 packets.

Total number of packets dropped from 500,000 to 220,000, reducing the amount of data transmission is about $40 \%$ of the original.

\section{Conclusion}

In order to effectively communicate the smart meter for electricity in real time, reliable measurement, analysis and design papers efficient, flexible, low-cost residential users access to the new structure of the data transmission network. In-depth knowledge of the relevant structural analysis and community needs, based on the proposed use of three new access system network topology, while ensuring access to coverage while improving the flexibility and scalability of the system, and can support underlying protocol conversion and the new IPv6 protocol.

On the other hand, for a wide range of smart meters large, huge problems collecting data presented SNMP-based transport protocol, the collected data classification metering device for multiple categories, and graded according to the degree of importance of the transmission. For a high degree of importance to join acknowledgment mechanism, and the introduction of compression and consolidation mechanism, to ensure efficient transmission, while greatly reducing the need for network bandwidth resources to achieve the reliability and economy while improving.

\section{References}

[1] Yu Yixin, Luan Wenpeng. Smart Grid Review[J]. Proceedings of the CESS, 2009, 29(34):1-8.

[2] Luan Wenpeng. Advanced metering system [J]. China Southern Power Grid Technology, 2009, 3(2): 6-10.

[3] Jin Zhigang, Liu Jia. Made power grid smarter by IOT[J]. Journal of the Hebei Academy of Science, 2011, 28(3): 5-9.

[4] Yu Yixin, Liu Bo, Luan Wenpeng. Nonintrusive Residential Load Monitoring and Decomposition Technology, SOUTHERN POWER SYSTEM TECHNOLOGY, 2013, 7(4):1-5.

[5] Lin Gongping, Wang Kaifu. Optical fiber communication technology in distribution automation system [J]. Automation of Electric Power Systems, 1998, 22(8): 64-66.

[6] Wang Sitong, Zhou Hui, Yuan Ruiming, et al. Concept and Application of Smart Meter [J]. Powers system technology,2010,29(4):17-23

[7] Wang Qian, Yang Jinling. Intelligent electric instrument network communication and communication protocol research [J]. Shandong Electric Power Technology, 2012,19(1):41-44.

[8] Kang Lun. ZigBee-based smart meter system design [D]. Hangzhou University of Electronic Science and Technology, Master Thesis. 2013.12. 